\title{
Acceleration of Anomalous Cosmic Rays: Solar Cycle Variation
}

\author{
József Kóta*t \\ University of Arizona, Tucson AZ 85721-0092, USA \\ E-mail: kota@lpl.arizona.edu
}

\begin{abstract}
The recent 2007-2009 weak and long solar minimum offers unique possibilities for gaining new insights into the physics of cosmic-ray transport. This cycle was distinctly different from earlier 'typical' cycles. Galactic and anomalous cosmic rays (GCRs and ACRs), whose solar-cycle variation do typically track each other quite closely (with proper scaling factors) behaved quite differently: GCRs reached record-high level in 2009, while ACR fluxes remained below their maximum 22 years earlier, suggesting that the source spectrum of the accelerated ACRs at the TS was lower. We may anticipate a similarly quiet solar minimum with the important difference, that the Sun has reversed its magnetic polarity. The present work focuses on possible effects on ACR acceleration connected with particle drifts in the large-scale heliospheric magnetic field (HMF). The roles of the pole-to-equator electric potential and the wavy heliospheric current sheets (HCS) are addressed. Drift effects due to the wavy HCS are simulated by employing a 2D axially symmetric 'hoop-model', which keeps computations at a comfortable level, while still captures the robust effects of the 3D wavy HCS. The preliminary results presented here are qualitative and are not yet intended for quantitative agreement with observations.
\end{abstract}

36th International Cosmic Ray Conference -ICRC2019-

July 24th - August 1st, 2019

Madison, WI, U.S.A.

* Speaker. 


\section{Introduction}

The recent 2007-2009 unusually weak solar minimum offers unique possibilities for gaining new insights into the physics of cosmic-ray transport. This cycle was distinctly different from earlier 'typical' cycles. One of its tantalizing features was that galactic and anomalous cosmic rays (GCRs and ACRs), whose solar-cycle variation do typically track each other quite closely (with proper scaling factors) behaved quite differently: GCRs reached record-high level in 2009 [9], while ACR fluxes remained below their maximum 22 years earlier [2]. We may anticipate a similarly quiet solar minimum [3], [4] with the important difference, that the Sun reversed its magnetic polarity.

GCRs are an important element of space-weather, they are also often used as a proxy of solar activity in the past. The study of cosmic rays during the last unusual solar minimum is relevant to the mechanism of the solar activity cycle. ACRs produced in our heliosphere provide a unique tool to explore the acceleration and transport of cosmic rays.

The disparity between GCRs and ACRs suggests that the production of ACRs at the termination shock (TS) was lower than in previous cycles. The cause of the apparent disparity between GCRs and ACRs is probably a combination of various effects. Moraal \& Stoker [5] were the first to propose a natural interpretation pointing out that the flux of ACRs reaching Earth critically depends on the maximum energy of acceleration. GCR intensity was high because of the easier diffusion in he weaker HMF results in less modulated GCR spectrum at Earth. This faster diffusion does, however, also reduce the rate of acceleration and the maximum energy of ACRs.

Other possible causes include but are not limited to dynamical changes in the location and strength of the TS, change in the pick up ion density providing the seed population, as well as possible drift effects connected with the large-scale structure of the HMF and its current sheet (HCS). The present work focuses on these drift effects. Throughout this paper, we work under the paradigm that ACRs are accelerated by diffusive shock acceleration (DSA) at the TS [6].

\section{Key Observations and their Implications}

The 2007-2009 solar minimum was unusual in more ways than one. The strength of the HMF decreased by a significant factor [7], and so did the density and ram pressure of the solar wind [8]. Mewaldt [9] reported that the 180-230 MeV/n GCR Oxygen observed by ACE reached record-high level in 2009. Record-setting high levels of GCRs were observed in ground-based neutron monitors as well. In contrast to GCRs, ACRs did not exceed their 1987 level two cycles earlier [2].

The HCS remained remarkably tilted, and flattened only in 2009. Thie flattening of the HCS was followed by a surge of the already high GCR intensity. Similar surge was seen in the ACR fluxes, indicating a lessening modulation for both GCRs and ACRs in the inner heliosphere. The cause of the disparity had likely been in the acceleration ACRs at the TS. GCRs undergo only smaller re-acceleration at the TS.

GCRs were only moderately modulated during subsequent (2015) solar maximum. Neutron monitor fluxes did not sink below their long-time 22-year average (including maxima as well as minima). Opposed to its usual $1 \mathrm{GV}$ value at solar maxima, the commonly used modulation potential, $\Phi$, has barely reached $700 \mathrm{MV}$ in 2015 [3]. The current level of GCRs are below the 2019 
intensity maximum but do already exceed the previous intensity maxima recoreded in the $A>0$ polarity cycle. Hence we can anticipate a similarly quiet next solar minimum this time with the opposite polarity state of the Sun.

The 2009 record-high GCR intensity was most likely the result of the weaker than usual strength of the IMF together with the flattening of the HCS in 2009. Beside this there is a number of other effects that may play a role.

- One possible cause is the slower rate of acceleration at the TS [5]. ACRs reaching the Earth must originate from higher energies at the TS (they lose energy on their path to Earth), thus the observed ACR flux at ACE is sensitive to the maximum energy which ACRs can gain at the TS. Lower rollover energy of ACRs results in lower fluxes at Earth.

- The TS is a near perpendicular shock, ions drift along the shock in the acceleration process, hence it is natural to expect drift effects, which may lead to charge dependence. Expectations for the approaching solar minimum may well differ from those in 2008-2009.

- A further plausible cause may rise from dynamical effects due to the decrease of the solar wind ram pressure. The shock may move inward and its strength may weaken. The role of pickup ions may become increasingly important. Recent New Horizon observations between 20-40 AU indicate that the heating of PUIs can be quite significant [10].

- Though it seems unlikely that we will go into a grand minimum, the last cycle resembles previous grand minima [11]. For instance, cosmogenic nuclei $\left(\mathrm{C}^{14}\right.$ and $\left.\mathrm{Be}^{10}\right)$ records suggest that measurable modulation of GCRs took place during the Maunder minimum [12]. The study of weak solar cycles may assist a better understanding of the past cycles.

\section{Simulation Models of ACR acceleration and transport}

We carry out numerical modeling under the paradigm that ACRs are accelerated at the solar wind TS [6], by DSA described by the robust Parker-equation [13]:

$$
\frac{\partial F}{\partial t}=\frac{\partial}{\partial x_{i}}\left(\kappa_{i j} \frac{\partial F}{\partial x_{j}}-V_{i} F\right)+\frac{\partial V_{j}}{\partial x_{j}} \frac{\partial F}{\partial \ln p^{3}}+Q
$$

where $F=p^{3} f\left(x_{i}, p, t\right)$ with $f$ standing for the phase space density in space, $x_{i}$ and momentum $p$. $V_{i}$ is the solar wind velocity, while the $\kappa_{i j}$ is the full diffusion tensor, including an anti-symmetric component, which describes regular motion in the large scale HMF, and which is responsible for particle drifts. Here we consider Eq.(3.1) on a finite grid of radius, latitude, and the logarithm of momentum, $\ln p$.

Shock acceleration produces power-law spectra in a natural way. The spectral exponent depends on the compression ratio at the shock up until a roll-over energy when spectra break down. This occurs when the rate of acceleration becomes slow and cannot overcome the rate of cooling or other losses.

Examples depicted in Figure 1 demonstrate that either faster diffusion or weaker shock results in softer spectrum at the TS and lower intensities inside the the TS. These simple simulations are carried out for spherical geometry without the inclusion of drift effects. The left panel shows and example where the diffusion coefficient is doubled, the right panel shows an example when the shock strengths is weakened from the original 3 to 2.5. The spectrum accelerated ACRs softens in both cases. 

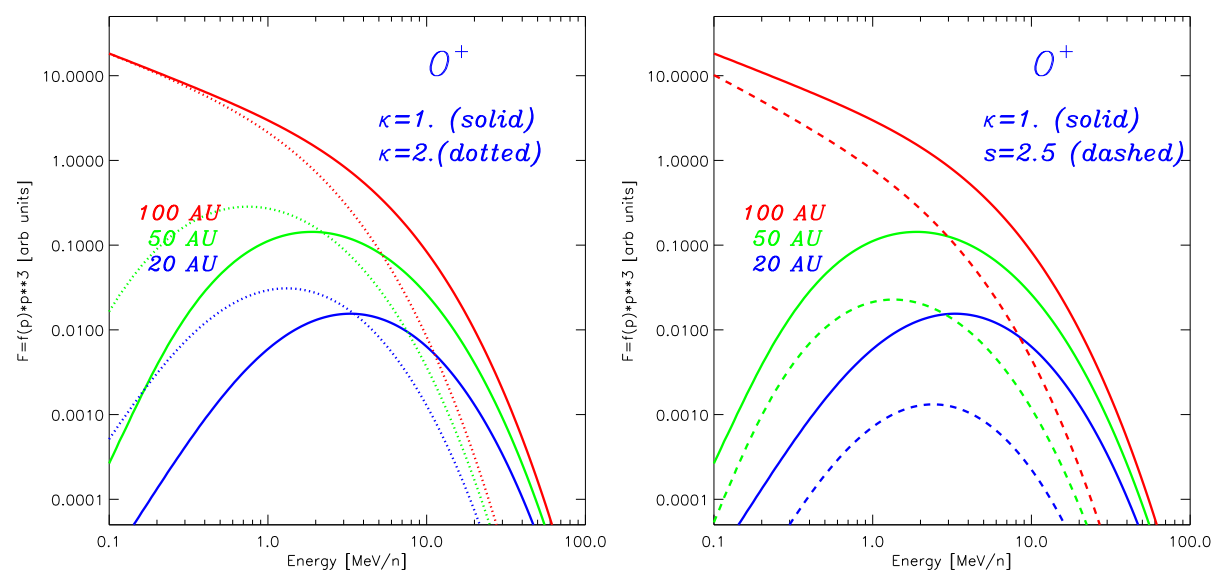

Figure 1: Illustrative examples, with fictitious parameters, showing that either faster diffusion or weaker shock result in lower cut-off energy in the ACR spectrum at the TS (red), leading to lower ACR fluxes inside the TS (green/blue). On the left panel the diffusion coefficient, $\kappa$, is increased by a factor of 2 , on the right panel the shock strength, $s$ is decreased from $s=3$. to $s=2.5$ (solid and dotted/dashed lines, respectively).

\subsection{The role of the equator-to-pole electric potential}

The TS is a highly perpendicular shock where drifts play essential role in the acceleration process: ions drift along the shock in the direction of the $V \times B$ electric field. Jokipii [14],[15] pointed out that the potential-difference between the pole and equator should set a natural limit for the energy-gain of ACRs accelerated at the TS.

It is instructive to consider a 1D planar case, where the plasma flows in the $+x$ direction, while the magnetic field is in the $x-y$ plane. Then one can show that the average displacement in $\operatorname{logarithmic~momentum,~} \ln p$ and in spatial $z$ direction are

$$
\langle\Delta \ln p\rangle=\left\langle\ln \left(p / p_{0}\right)\right\rangle=\frac{V_{1}-V_{2}}{3 V_{2}} \quad \text { and } \quad\langle\Delta z\rangle=\left\langle z-z_{0}\right\rangle=\frac{\kappa_{x z, 1}-\kappa_{x z, 2}}{V_{2}}
$$

where indices 1 and 2 refer to upstream and downstream values, respectively. Equations (3.1) tell, after short algebra, that the mean momentum gain and mean spatial displacement are closely connected. For nearly perpendicular shocks the mean energy gain should be in the range of the the potential difference along the TS, yielding $\Delta E \approx Z e \Delta \Phi$, where $Z e$ is the charge of the charge of the particle. Hence multiply charged ACRs can gain more energy [15].

It is straightforward to see that, under the near steady-state conditions of a flat HCS, the poleto-equator potential, $\phi$, is proportional to the total magnetic flux in one hemisphere. For for steady corotating HMF. $B_{i}$ is in the direction of the $V-\Omega \times r$ vector, so the $B \times V$ electric field equals

$$
B \times V=B \times(\Omega \times r)
$$

the integral of which from the 'pole to equator gives

$$
\phi=\frac{\Omega}{2 \pi v} \int B . d A
$$

We can expect that the weaker magnetic field should result in lower rollover energy and hence fewer high-energy ACRs. Figure 2 shows the schematic view of of ACR acceleration during solar 

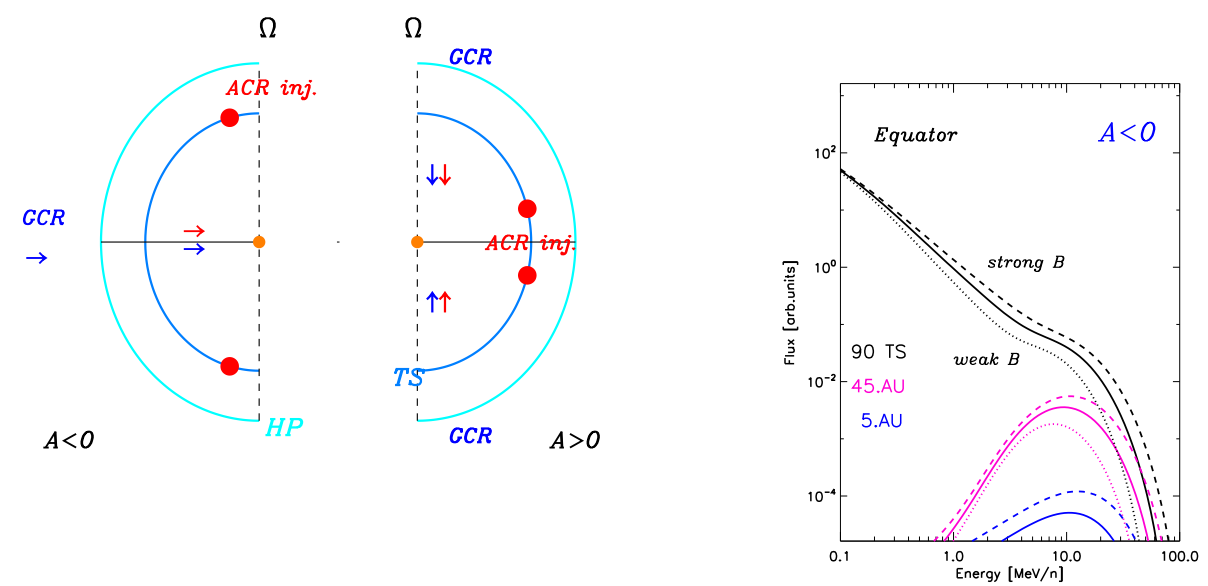

Figure 2: Schematic view of ACR acceleration during solar minima. The divided left panel indicates the direction of drifts arrows) and the preferential sites of ACR acceleration (red blobs, see text). The right panel demonstrates that stronger HMF (dashed line) yields harder ACR spectrum at the TS while a weaker HMF (dotted line) gives softer spectrum.

minima. The left panel is divided, the two sides refer to the $A<0$, and $A>0$ polarity states. For $A<0$ both GCRs and ACRs drift inward along the near equatorial HCS. Seed particles of ACRs have to be injected at the polar region, particles injected at lower latitudes cannot gain high energies. Conversely, for $A>0$ cosmic rays drift inward through the polar regions, so ACRs have to be have to be injected at low latitudes. The right panel shows a simulation illustrating that stronger HMF (dashed line) yields higher, the weaker field gives lower rollover energies of the ACR spectrum resulting in higher/lower ACR flux at Earth.

\section{2 'Hoop' model of the 3D helospheric currant sheet}

The wavy HCS is an important element of cosmic rays transport. Particles can drift fast along the HCS, and the direction of drift reverses with the polarity change of the Sun. The wavy HCS is an inherently 3-D structure whose precise incorporation requires 3-D codes, that are typically more cumbersome than 2-D codes, and they are also prone to stability problems. While 3-D codes are doable [17],[18], the more transparent 2-D codes should be sufficient for our purpose of identifying the robust features.

Since potential importance particle drift was recognized [19], a great deal of work has been devoted to its role in cosmic-ray transport. Drift velocity is derived from the anti-symmetric component of the "full" diffusion tensor, which may be reduced by turbulent fluctuations, the precise reduction is, however, still controversial [20],[21],[22], [23].

The South African School developed a series of 2-D codes emulating 3-D effects of the HCS. This approach has been applied for the description of solar modulation [24],citeMSP2014. Here we use a 2-D axially symmetric model, that emulates the 3-D waviness of the HCS taking a different approach [27], [23]. This is a 'hoop model' similar in concept to that suggested by Drake [26] to simplify a complex heliospheric structure. The Parker spiral is tightly wound in regions 

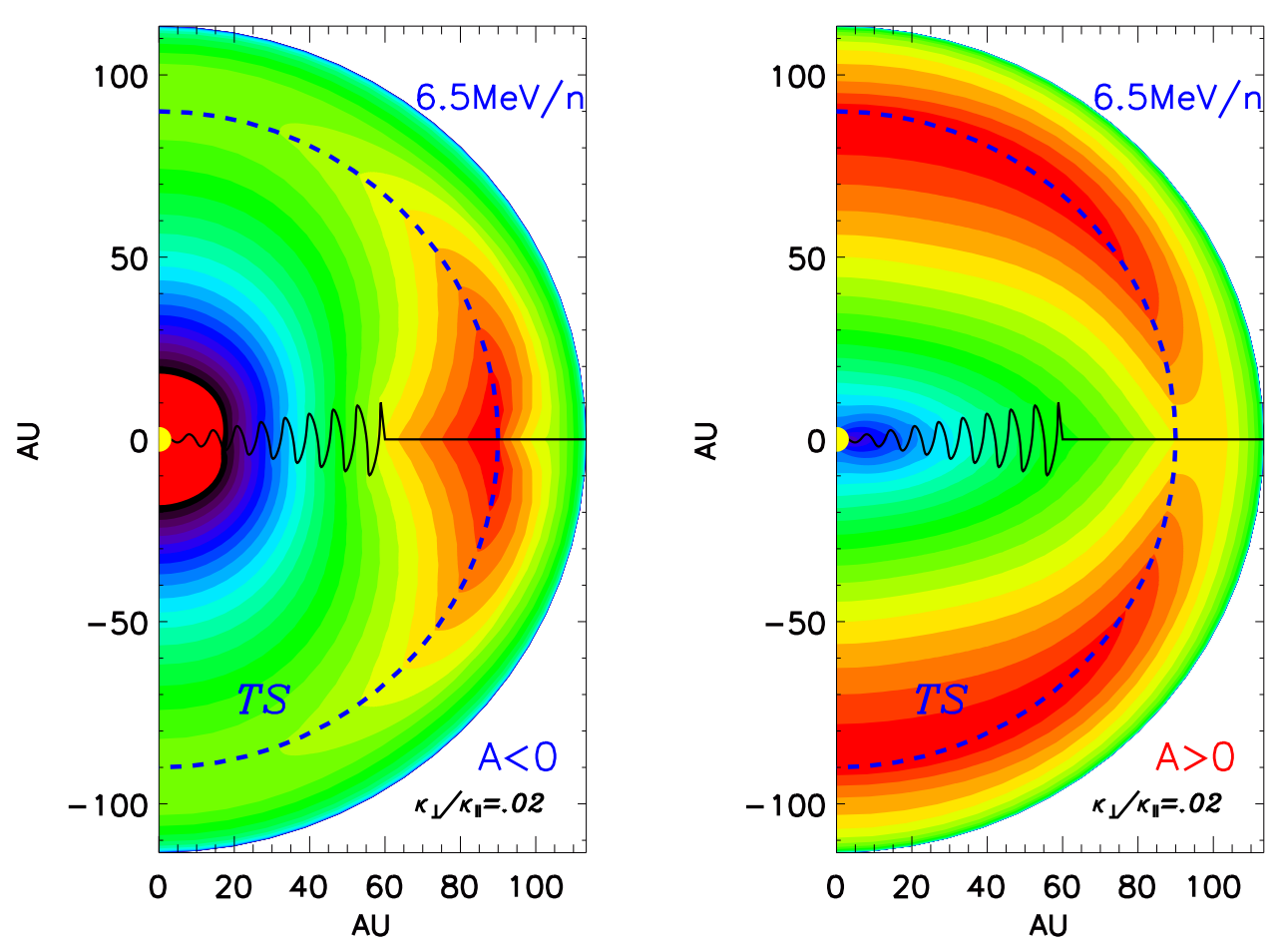

Figure 3: Sample contour-plots of ACR intensity in a kinematical hoop model during two consecutive solar minima. The contours show the well known polarity dependence in the meridional distribution of ACRs at solar minima. Black line indicate the momentary HCS that is carried out radially by the solar wind.

beyond 10-20 AU; hence parallel diffusion is mostly in the axial direction and both the radial and latitudinal diffusions are dominated by the otherwise smaller perpendicular component, $\kappa_{\perp}$. We construct a 'hoop' model, where the spiral field is substituted by expanding hoops that are carried out radially by the supersonic solar wind. The waviness if the HCS appears as timedependence in the magnitude and polarity of the axial $B$. We still use an anisotropic diffusion, taking $\kappa_{r r}=\kappa_{\perp}+\left(\kappa_{\|}-\kappa_{\perp}\right) * \cos ^{2}(\psi)$ where $\kappa_{\|}$and $\kappa_{\perp}$ stand for the parallel and perpendicular diffusion coefficients, while $\psi$ is the garden-hose angle of the Parker spiral. The polar field is modified according the way of [22]. The structure of the inner heliosheath, which is overly simplified now, will later be upgraded along the line of Drake's hoop model [26].

Figure 3 illustrates how this hoop model simulates the solar cycle variation of singly ionized Oxygen ACRs. Samples show the the meridional distribution of ACRs at solar minima. The waviness of the HCS is switched on at $t=0$, so these distribution of ACRs display 'typical' solar minimum conditions for the respective polarity states.

Figure 4. depicts simulated time variations of GCRs and ACRs. The left panel shows GCRs during an $A<0$ period between two consecutive solar maxima. The tilt of the HCS and the strength of the magnetic field are changed in a way resembling to the last cycle. The model is preliminary, parameters are largely fictitious, so we did not strive for quantitative agreement. The simulation shows the observed surge of GCRs at the flattening of the HCS in 2009. 

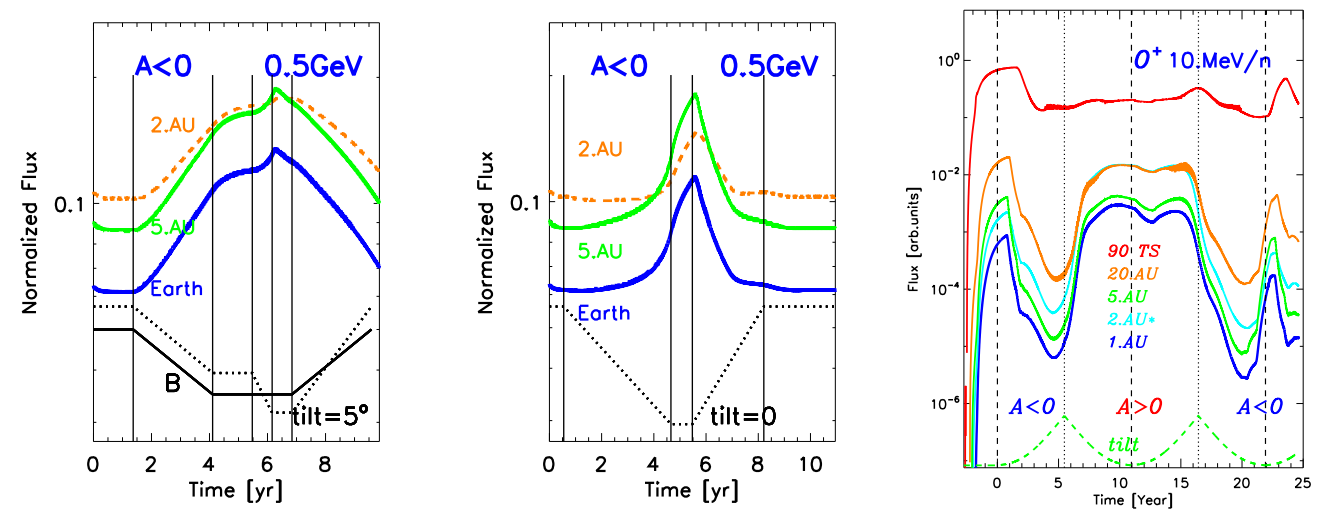

Figure 4: Preliminary results simulating the variation of $0.5 \mathrm{GeV}$ GCRs during a period resembling the last solar cycle (left panel). The magnetic field strength and the tilt of the HCS changes continuously (shown at bottom). The medium panel shows a simulation when the HCS tilt angle is the single parameter to change. The right panel displays the 22-year cyclic variation of dingly charged Oxygen ACR in response to the variation of the HCS tilt only.

The medium panel of Fig.4 displays a simulation where the tilt angle is the single parameter to change. The characteristic peaky structure is reproduced but we cannot expect quantitative agreement, this serves only as a numerical experiment only.

The right panel shows a preliminary simulation of O+ ACRs during a full 22 year cycle containing both $A<0$ and $A>0$ periods; the tilt angle changes continuously and flips from one polarity to the other. This is again a simulation where the tilt angle is the single parameter to change from 0 to 90 degrees and back as shown by the green curve at the bottom of the panel.ACRs follow the change of the tilt with some delay due to the combination of times for ACR transport and the times it takes the solar wind to to carry the HCS out to larger radial distance. The apparent 'asymmetry' between $t=0$ and $t=22$ years happens since $t=0$ is preceded by 3 years of flat HCS,

\section{Summary}

The disparity between GCRc and ACRs during the 2007-2009 solar minimum may have been caused by a several factors or, most likely, the combination of those. For instance, the lower faster diffusion in the weaker $B$ field can lower the maximum energy of ACR, which gives a natural explanation of the lower ACR flux at Earth [5]. Even more, we find that a weaker $B$ will lower the maximum energy without evoking faster diffusion.

Among the possible causes we focused on drift effects with special interest of the role of the wavy HCS. Our main goal is to experiment with a 2D axially symmetric 'hoop' model of the 3D wavy HCS. While this hoop-model has some limitation it has several advantages that, we believe, outnumber the limitation. Though our model is $2 \mathrm{D}$ and axially symmetric, it captures the most essential effects of the HCS, and can readily be extended to include other factors (e.g. multiple charges, dynamical effects, or even 27-day variations, etc.)

Though it not our primary science goal, the investigation of disparity between GCRS and ACRs can advance our understanding of the modulation of GCRs during extended periods of very 
low solar activity, isuch as the period of the Maunder minimum, when the Sun was very quiet, yet, according to cosmogenic nuclei, GCR intensity was still modulated. It is conceivable that the wavy HCS was the main driver of modulation during Grand minima.

\section{Acknowledgements}

The author benefited from discussions with Professor J.R. Jpkipii. This work was supported by NASA LWS program under grant NNH15ZDA001N.

\section{References}

[1] R. Mewaldt, A.J. Davis, K.A. Lave, et al., Astrophys. J.. 723 (2010) 1

[2] R.A. Leske, A.C. Cummings, R. Mewaldt, \& E.C. Stone, Space Sci. Rev., 176 (2013) 253

[3] I.G. Usoskin, A. Gil, G.A. Kovaltsov et al., J. Geophys. Res., 122 (2017) 3875

[4] R.A. Leske et al., AGU Fall Mtg., SH54A (2017)

[5] H. Moraal \& P.H. Stoker, Geophys. Res. Lett, 115 (2010) 12109

[6] M. Pesses, J.R. Jokipii, \& D. Eichler, Astrophys. J., 246 (1981) 85

[7] E.J. Smith \& A. Balogh, Geophys. Res. Lett., 35 (2008) L22103

[8] D.J. McComas, R.W. Elbert, \& H.A. Elliot, Geophys. Res. Lett., 35 (2008) L18103

[9] R. Mewaldt, et al., ACE News, 136, (2010)

[10] D.J. McComas, et al., Astrophys. J. Suppl., 233 (2017) 14

[11] J.W. Harvey, Space Sci. Rev., 176 (2013) 47

[12] J. Beer, K.G. McCracken, J. Abreu, U. Heikilla, \&F. Stenhilber, Space Sci. Rev., 176 (2013) 89

[13] E.N. Parker, Planet. Space Sci., 13 (1965) 9

[14] J.R. Jokipii, Astrophys. J.. 255 (1982) 716

[15] J.R. Jokipii, Astrophys. J.. 466 (1996) 47

[16] J. Kóta, in Astronum 10 ASPC bf 444 (2010) 48

[17] J. Kota \& J.R. Jokipii, Astrophys. J.. 265 (1983) 573

[18] J. Kota \& J.R. Jokipii, Geophys. Res. Lett., 18 (1991) 1797

[19] J.R. Jokipii, E.H. Levy, \& W.B. Hubbard, Astrophys. J.. 213 (1977) 861

[20] S. Dalla, M.S. Marsh, J. Kelly, \& T. Laitinen , J. Geophys. Res, 118 (2013) 5979

[21] N.E. Engelbrect, et al., Astrophys. J., 841 (2017) 107

[22] J.R. Jokipii, \& J. Kota, Geophys. Res. Lett.. 16 (1989) 1

[23] J. Kóta, JPhCS, 767 (2016) id:012014

[24] M.S. Potgieter \& H. Moraal, Astrophys. J., 294 (1985) 425

[25] M.S. Potgieter, Living Rev. P.S., 10 (1985)

[26] J.H. Drake, M. Swisdak, \& M. Opher, Astrophys. J.. 709 (2015) 963

[27] J. Kóta, Space Sci. Rev., 176 (2013) 391 